\title{
First steps towards time-resolved BioXAS at room temperature: state transitions of the manganese complex of oxygenic photosynthesis
}

\section{Michael Haumann, ${ }^{\text {a* }}$ Pavel Pospíšil, ${ }^{b}$ Markus Grabolle, ${ }^{a}$ Claudia Müller, ${ }^{a}$ Peter Liebisch, ${ }^{a}$ V. Armando Solé, ${ }^{c}$ Thomas Neisius, ${ }^{c}$ Jens Dittmer, ${ }^{b}$ Lucia luzzolino ${ }^{b}$ and Holger Dau ${ }^{a}$}

\author{
${ }^{a}$ Freie Universität Berlin, FB Physik, Arnimallee 14, D-14195 \\ Berlin, Germany, ${ }^{b}$ Philipps-Universität Marburg, FB Biologie, \\ Lahnberge, D-35032 Marburg, Germany, and ${ }^{C} E S R F, B P 220$, \\ 38043 Grenoble CEDEX, France. \\ E-mail: haumann@physik.fu-berlin.de
}

Structural changes and redox transitions at the metal atoms of the active site are essential for the understanding of the catalytic mechanisms of biological metalloenzymes. First steps towards studying these processes by time-resolved X-ray absorption spectroscopy on protein samples (BioXAS) are reported. Photosystem II (PSII) catalyses the light-driven oxidation of bound water molecules at a tetranuclear manganese complex yielding the molecular oxygen of the atmosphere. In this work, first time-resolved XAS results under quasi-physiological conditions (at room temperature, non-crystalline samples) on PSII are presented. Perspectives of time-resolved BioXAS are discussed.

Keywords: bioinorganic chemistry; EXAFS; manganese complex; metalloenzymes.

\section{Introduction}

The understanding of catalytic processes in biological macromolecules provides insights into the principles of life and is the basis for biomimetic and biotechnological developments. It is estimated that $25-50 \%$ of all enzymes are metalloenzymes (Hill et al., 1999). They contain protein-bound metal atoms which, inter alia, position and activate substrate molecules and serve as sources/sinks of electrons in redox reactions. Typically, the catalytic function involves structural and/or redox changes at the metal site. By protein crystallography and X-ray absorption spectroscopy (XAS) at cryogenic temperatures, normally the structure of a 'resting state' of the active site is determined. This statical picture, however, often does not yield information on the catalytic mechanism (Yachandra, 1995; Scott, 2000).

A promising new method for the analysis of changes of the metal site is XAS at room temperature. Under these 'quasi-physiological' conditions, enzymatic catalysis is active. In principle, the dynamics of substrate binding and structural and redox changes during the catalytic reactions can be studied in atomic detail, and variations of important parameters $(\mathrm{pH}$, substrate concentration, protein composition, temperature, duration of X-ray exposure etc.) are possible (Meinke et al., 2000). Only the high X-ray intensity provided by thirdgeneration synchrotron sources in combination with rapid-scan capabilities (Solé et al., 1999) facilitates previously impossible timeresolved investigations of the metal sites of metalloenzymes.

XAS investigations on protein samples are particularly difficult. Owing to the relatively low metal concentration of only about $1 \mathrm{mM}$ that can be achieved in these samples, X-ray absorption spectroscopy cannot be performed in absorption mode. Instead, the excited X-ray fluorescence is detected. Fluorescence detection is, on the one hand, advantageous in providing well resolved spectra with low pre-edge background. On the other hand, fluorescence detection prohibits the use of techniques such as dispersive XAS which allow for the rapid measurement of complete XAS spectra using broadband X-rays. Alternatively, XAS spectra may be sampled 'point-by-point'. However, large amounts of protein samples and sophisticated averaging strategies need to be used to obtain high-quality XAS spectra of metalloenzymes.

Here, we report first time-resolved XAS results on the manganese (Mn) complex of a photosynthetic metalloenzyme, photosystem II (PSII), at room temperature. PSII is a multi-subunit protein-cofactor complex found in the thylakoid membrane of higher plants and cyanobacteria. It catalyses the light-driven oxidation of two water molecules to molecular oxygen. This process is of fundamental biological importance as it provides most of the oxygen in the atmosphere (Haumann \& Junge, 1996; Renger, 2001). The catalytic function of PSII takes place at a protein-bound tetranuclear manganese complex. Water oxidation by PSII is a five-step process. Excitation of the enzyme by a short flash of light leads to the abstraction of one electron from the Mn complex. By successive application of light flashes, the Mn complex becomes increasingly oxidized. The formal redox states are named $\mathrm{S}_{0}, \mathrm{~S}_{1}, \ldots, \mathrm{S}_{4}$. Each of the four electron abstractions induces a $S_{i} \Rightarrow S_{i+1}$ transition until, finally, $S_{4}$ is reached. The $S_{4}$ state is rapidly converted to $S_{0}$ (without additional light flash) and dioxygen is released, thereby closing the reaction cycle (Kok et al., 1970).

Previously, XAS results on the manganese complex in its $S_{1}$ state have been obtained at cryogenic temperatures [for reviews see Penner-Hahn (1999), Robblee et al. (2001), Dau et al. (2001)]. The results are indicative that all the transitions between S-states are coupled to oxidation state changes (Ono et al., 1992; Roeloffs et al., 1996; Iuzzolino et al., 1998; Messinger et al., 2001) and to EXAFSdetectable structural changes (Dau et al., 1998; Liang et al., 2000; Haumann et al., 2002) (EXAFS = extended X-ray absorption fine structure). These changes are, so far, only insufficiently characterized and understood.

In the present investigation, the redox transitions of the Mn complex were initiated by the application of short flashes of light to PSII samples exposed to the X-ray beam of the undulator beamline ID26 at the European Synchrotron Radiation Facility (ESRF, Grenoble, France). XAS scans of only seconds duration were performed. The current status and future impact of the method of time-resolved room-temperature BioXAS is discussed.

\section{Materials and methods}

X-ray absorption measurements were carried out at the undulator beamline ID26 of the ESRF. By scanning of a Si220 crystal monochromator, the X-ray fluorescence was excited; it was monitored by a photodiode (active area $\sim 4 \mathrm{~cm}^{2}$ ). Thin chromium and aluminium filters in front of the photodiode suppressed scattered X-rays and light. PSII samples were placed in plain air. One scan per sample was performed on dark-adapted samples (X-ray spot size $\sim 0.5 \times 1 \mathrm{~mm}$, photon flux $\sim 10^{12} \mathrm{~s}^{-1}$ ). For further details see Signorato et al. (1999), Solé et al. (1999), Meinke et al. (2000).

Flash-excitation of the samples was performed at ID26 either by xenon-flash lamps (>410 nm, FWHM $10 \mu \mathrm{s}, \sim 10 \mathrm{~mJ}$ per flash) or by a frequency-doubled, Q-switched Nd-YAG laser (Quantel Brilliant, FWHM $5 \mathrm{~ns}, 532 \mathrm{~nm}, \sim 150 \mathrm{~mJ}$ per flash). The light flashes illumi- 
nated both sides of the sample simultaneously, synchronized with data acquisition by appropriate trigger electronics.

We performed two types of experiments. (i) Time scans were carried out at a fixed X-ray energy and the X-ray fluorescence intensity was measured as a function of time. A rapid beam shutter ( $t_{\text {open }} \simeq 10 \mathrm{~ms}$ ) opened about $0.2 \mathrm{~s}$ before the start of data collection. (ii) XAS scans using the rapid-scan mode (synchronous scan of undulator gap and monochromator) of ID26 were performed after distinct numbers of light flashes. Flashes were applied to the sample, then the rapid beam shutter was opened ( $0.2 \mathrm{~s}$ after the last flash), and data collection started $\sim 0.2 \mathrm{~s}$ later. All experiments were carried out at room temperature.

Data evaluation was carried out as described elsewhere (Schiller $e t$ al., 1998; Iuzzolino et al., 1998). Energy calibration was facilitated by using the narrow pre-edge peak of the simultaneously measured $\mathrm{KMnO}_{4}$ absorption as a standard (Schiller et al., 1998; Meinke et al., 2000). The Mn $K$-edge energy was determined using the integral method' outlined by Dittmer et al. (1998).

Oxygen-evolving PSII-enriched membrane particles and PSII membrane multilayers with the Mn complex in the $\mathrm{S}_{1}$ state were prepared as previously described (Iuzzolino et al., 1998; Schiller \& Dau, 2000). The samples contained $200 \mu \mathrm{M}$ of the artificial electron acceptor PPBQ (Schiller et al., 1998; Iuzzolino et al., 1998; Dittmer \& Dau, 1998). Each sample contained about $0.14 \mathrm{mg}$ of chlorophyll and $\mathrm{Mn}$ at a concentration of about $1 \mathrm{mM}$.

\section{Results}

\subsection{Time-resolved BioXAS to follow oxidation state changes}

The X-ray energy was set to a fixed value in the region of the Mn $K$-edge. Fig. 1( $a$ ) shows a time scan of the X-ray fluorescence excited at $6549.5 \mathrm{eV}$. After onset of X-ray irradiation, the fluorescence intensity increases within only $3 \mathrm{~min}$ to a stable value. This increase reflects the downshift of the Mn $K$-edge owing to the photoreduction of the Mn complex (Meinke et al., 2000). Before and after 3 min of X-ray irradiation of the sample (arrows in Fig. 1), the amount of photoreduction was verified by recording Mn $K$-edge spectra [X-ray absorption near-edge structure (XANES)] within only $1 \mathrm{~s}$ using the rapid-scan mode of ID26. The initially collected XANES spectrum (inset in Fig. 1a) is typical for the Mn complex in its $\mathrm{S}_{1}$ state (solid line) whereas the spectrum collected after 3 min of X-ray irradiation is typical for samples containing $\mathrm{Mn}^{2+}$. The spectra reflect the transition from the $\mathrm{Mn}(\mathrm{III})_{2} \mathrm{Mn}(\mathrm{IV})_{2}\left(\mu_{2}-\mathrm{O}\right)_{4-6}$ complex of PSII to mostly $\mathrm{Mn}^{2+}$ in a hexaquo environment (Pospísil et al., 2001). The $\mathrm{Mn}^{2+}$ formation is due to the rapid reduction of the $\mathrm{Mn}$ atoms by numerous radicals generated in the sample by the high X-ray flux $\left(\sim 10^{12}\right.$ photons s $\left.^{-1}\right)$ (Dau et al., 1997).

From $\mathrm{Mn} K$-edge spectra recorded after increasing times of X-ray irradiation, the $K$-edge energies were determined. They are shown as a function of the irradiation time in Fig. 1(b). The Mn $K$-edge energy decreases at about the same rate $\left(\sim 1 \mathrm{~min}^{-1}\right)$ as determined for the fluorescence increase by the time scan at fixed X-ray energy (Fig. 1a). The Mn $K$-edge energies of the Mn complex were compared with those measured for various Mn oxides (Dittmer et al., 1998) (inset in Fig. $1 b$ ), yielding the time dependence of the mean oxidation state of the Mn complex in PSII (second $y$-scale in Fig. $1 b$ ).

In summary, oxidation-state changes of the Mn complex owing to photoreduction during X-ray exposure were time-resolved using either time scans at fixed excitation X-ray energies or a series of rapid XANES scans. Both experimental approaches facilitate the timeresolved investigation of oxidation-state changes at the active-site metal centre in proteins. Application of the rapid-scan technique is currently limited to relatively slow processes $\left(t_{1 / 2}>5 \mathrm{~s}\right)$ whereas there are no principle limitations with respect to the time resolution of the alternative time scan method.

\subsection{Time-resolved BioXAS to follow structural changes}

The energy of the exciting X-rays was fixed to a distinct value in the region of the Mn $K$-edge (around $6545-6660 \mathrm{eV}$ ). Then, a train of five xenon flashes was applied to PSII samples exposed to the X-ray beam and, simultaneously, the time-course of the X-ray fluorescence intensity was recorded (Fig. $2 a$, inset). Fig. 2(a) shows the levels of $\mathrm{X}$-ray fluorescence reached after 0 to 5 flashes (solid symbols). At all energies the fluorescence level decreases in response to the first and second flash and increases in response to the third flash. The relative extents of the changes, however, depend on the X-ray energies. A decrease of the fluorescence level is anticipated for a shift of the Mn

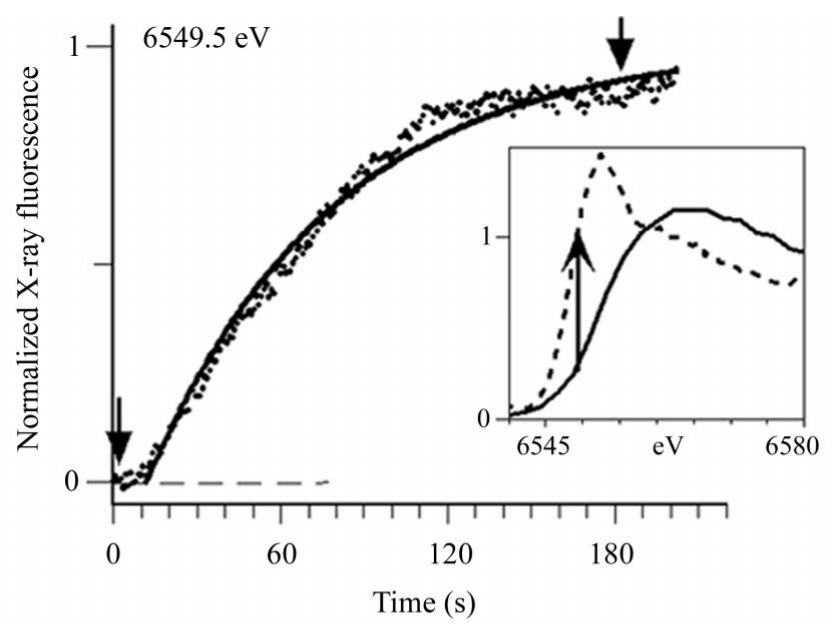

(a)

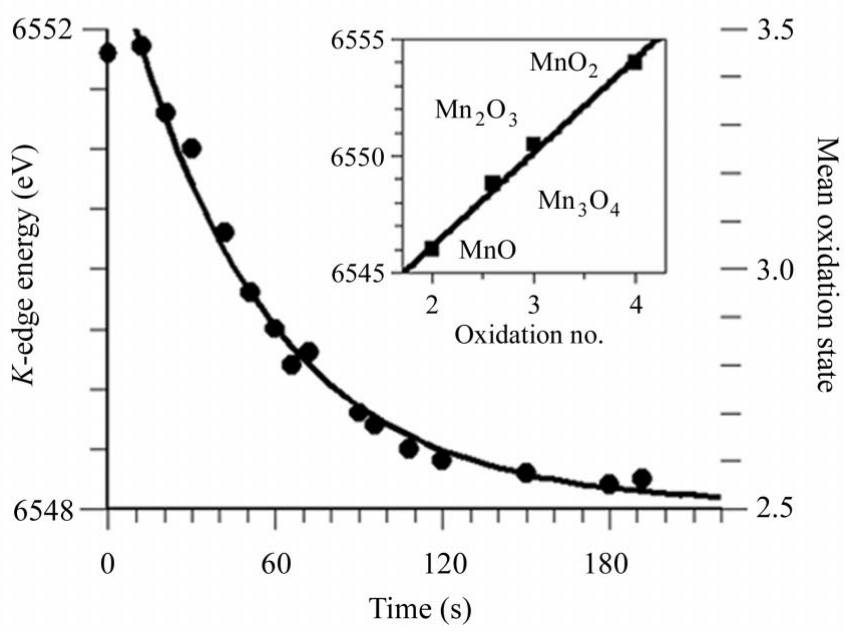

(b)

Figure 1

(a) Time scan of the X-ray fluorescence from the Mn complex of PSII for an excitation energy of $6549.5 \mathrm{eV}$ monitored at room temperature. Solid line: a single-exponential simulation with a rate constant of $0.9 \mathrm{~min}^{-1}$. The arrows indicate the times at which the XANES spectra (inset) were recorded. Inset: $\mathrm{Mn}$ XANES spectra after $0.5 \mathrm{~s}$ and $3 \mathrm{~min}$ of X-ray exposure. The arrow indicates the increase in fluorescence intensity at $6549.5 \mathrm{eV}$. (b) Time course of the $\mathrm{Mn} K$-edge energy. Energy values were derived from XANES scans like the ones shown in the inset of $(a)$. Inset: $K$-edge energies of various Mn oxides. The straight line was used to relate the $K$-edge positions shown in the main trace (second $y$-scale) to Mn oxidation states. 

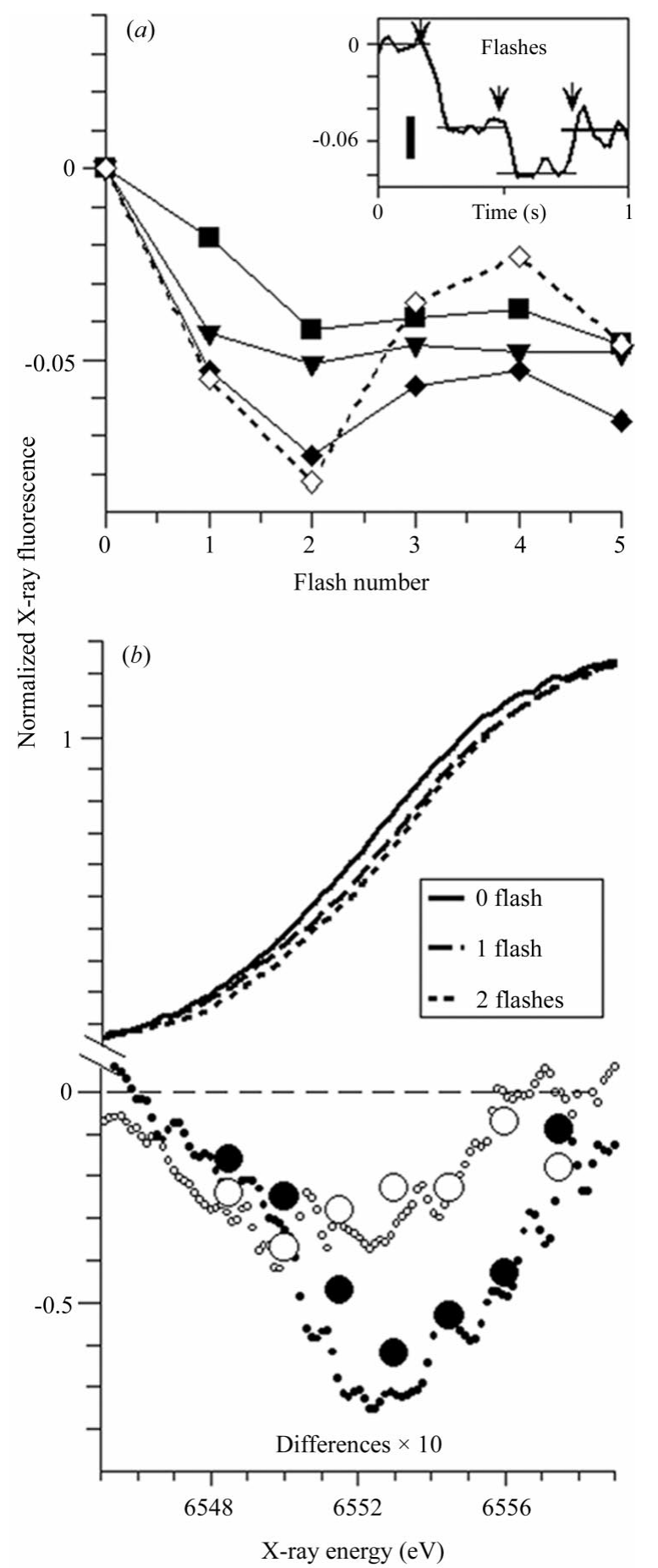

Figure 2

(a) X-ray fluorescence intensities observed after 0 to 5 xenon flashes (solid symbols) for selected excitation energies (- $6548.5 \mathrm{eV}$, $6551.5 \mathrm{eV}$, $6556.0 \mathrm{eV})$. The open diamonds $(\diamond)$ represent a flash pattern of fluorescence intensities obtained from ns laser-flash excitation at an X-ray energy of $6551.5 \mathrm{eV}$. The inset shows the time course of the fluorescence intensity recorded for excitation at $6551.5 \mathrm{eV}$ (xenon flashes, eight transients averaged, flash-burst artefacts removed and data smoothed for clarity); the vertical bar indicates the peak-to-peak noise level of a raw single-shot trace. (b) Upper part: Mn $K$-edge spectra from dark-adapted PSII (solid line, $\mathrm{S}_{1}$ state), after one (dashed line) or two (dotted line) light flashes. Lower part: difference between the dark spectrum and the one-flash spectrum (small solid circles); difference between one-flash and two-flash spectra (small open circles). Large symbols represent the fluorescence changes derived from traces as shown in (a); solid circles, first flash; open circles, second flash. For clarity, the differences in the lower part of $(b)$ have been scaled by a factor of ten.
$K$-edge to higher energies whereas an increase indicates a shift to lower energies.

Fig. 2(a) also shows changes of the fluorescence intensity induced by the application of ns laser flashes (open diamonds). Comparison of the fluorescence intensity changes induced by the application of xenon flashes or laser flashes at the same X-ray excitation energy, namely $6551.5 \mathrm{eV}$, reveals that a more pronounced oscillatory behaviour is induced by the laser flashes. This behaviour reflects the much shorter flash duration and higher intensity of the laser flashes resulting in the absence of 'double hits' (centres turning over twice upon the same flash) and in the reduction of 'misses' (centres not turning over upon a flash). Consequently, by the application of ns laser flashes, a higher enrichment and greater 'purity' of the respective S-states of the Mn complex is achieved after each flash.

X-ray absorption spectra were recorded at the $\mathrm{Mn} K$-edge (XANES) after the application of either zero or one or two xenon flashes to dark-adapted PSII samples using the rapid-scan mode of the beamline ID26. In the dark-adapted samples (zero flashes, Fig. $2 b$, upper part, solid line), the Mn $K$-edge energy was $6551.7 \mathrm{eV}$. The Mn $K$-edge was shifted to higher energies (Fig. $2 b$ ) by the first flash (dashed line) and by the second flash (dotted line), and to lower energies by the third flash (not shown). This behaviour is suggestive of an oxidation of the Mn complex (where the term 'Mn complex' denotes the $\mathrm{Mn}$ atoms plus their ligands) in response to the first and second flash; upon the third flash Mn reduction seems to occur. These oxidation/reduction reactions are expected for the Mn complex predominantly undergoing the redox transitions $S_{1} \Rightarrow S_{2}$ (first flash), $\mathrm{S}_{2} \Rightarrow \mathrm{S}_{3}$ (second flash) and $\mathrm{S}_{3} \Rightarrow \mathrm{S}_{0}$ (third flash) (Ono et al., 1992; Roeloffs et al., 1996; Iuzzolino et al., 1998).

The difference between the $\mathrm{Mn} K$-edge spectrum recorded after the first flash $\left(\mathrm{S}_{2}\right.$ state predominantly populated) minus the spectrum recorded without flash illumination $\left(\mathrm{S}_{1}\right.$ state) is shown in Fig. $2(b)$ (small solid circles). This difference spectrum roughly resembles the first derivative of the spectrum of the $S_{1}$ state. This behaviour indicates a shift in the edge position without major changes in the shape of the Mn $K$-edge. The difference between the Mn $K$-edge spectrum recorded after the second flash $\left(\mathrm{S}_{3}\right.$ state predominantly populated) minus the spectrum recorded after the first flash $\left(\mathrm{S}_{2}\right.$ state predominantly populated) is also shown in Fig. 2(b) (small open circles). This difference spectrum shows a maximum which is at an energy clearly below the inflection point of the $\mathrm{S}_{2}$-state spectrum; it differs from the first derivative of the $S_{2}$-state spectrum. This behaviour reveals that not only the edge position is at higher energies but also that the shape of the edge is different in the $S_{3}$ state. A different shape of the Mn $K$-edge in $\mathrm{S}_{2}$ and $\mathrm{S}_{3}$ has been previously observed using XAS measurements at cryogenic temperatures (Roeloffs et al., 1996; Iuzzolino et al., 1998; Messinger et al., 2001). These changes in the shape of the $K$-edge point to structural changes of the metal site.

The flash patterns of the fluorescence levels obtained at different X-ray energies (Fig. 2a) were used to 'extract' differences of spectra (Fig. $2 b$; solid circles, changes upon flash 1 ; open circles, changes upon flash 2). The difference spectra obtained by analysis of a series of time scans closely resemble the differences of the XANES spectra (Fig. $2 b$, lower part).

In summary, we collected time scans of the X-ray fluorescence intensity for each of seven selected excitation energies. Using this 'sampling of energies', changes in the X-ray absorption spectra were monitored with a time resolution of $20 \mathrm{~ms}$. The difference spectra obtained by the sampling technique resemble the difference spectra resulting from monochromator scans. Using the sampling approach, structural BioXAS investigations at $\mathrm{ms}, \mu$ s or even ns time resolution may become possible. 


\section{Discussion}

X-ray absorption spectroscopy under conditions where biological metalloenzymes are functional (e.g. at room temperature, non-crystalline samples) is a new approach towards the elucidation of oxidation state and structural changes at the metal site (Meinke et al., 2000). By starting the catalytic reaction using synchronizing trigger events (e.g. as provided by laser-flash excitation of photochemical reactions), time-resolved XAS measurements on changes at the metal site become feasible. In this work, we report first results obtained by using experimental strategies which have so far only rarely been applied in BioXAS.

(i) Time scans of the X-ray fluorescence and rapid-scan XAS with total scan times of only seconds duration allow for the time-resolution of oxidation state changes at the metal site. We investigated the reduction of the Mn complex in the $S_{1}$ state in response to X-ray exposure. At room temperature, photoreduction of the Mn complex is relatively fast (rate constant of $\sim 1 \mathrm{~min}^{-1}$ at an X-ray flux of $\sim 10^{12}$ photons s$^{-1}$ ). The $\mathrm{Mn}$ complex is reduced from an average oxidation state of initially 3.5 down to 2.5 ; the latter value indicates the presence of high amounts of $\mathrm{Mn}$ (II) in the sample. Inspection of the reduction time course reveals a small lag-phase of $\sim 10$ s duration. Whether such a lag phase is also present in higher oxidation states of the Mn complex is currently under investigation. During this time interval, seemingly the oxidation state of the Mn complex remains constant. Thus, by applying well adapted protocols, radiation damage of the Mn complex can be avoided even if measurements are performed at room temperature and using the high photon flux of an undulator beamline. However, rapid-scan capabilities are a prerequisite for the investigation of the structure of the (intact) Mn complex at room temperature. They allow for XAS scans (XANES plus EXAFS region) of less than $10 \mathrm{~s}$ duration. A reasonable signalto-noise ratio for the quantitative analysis of EXAFS spectra can be obtained by averaging of $\geq 20$ scans of 10 s duration (Haumann et al., 2001, 2002).

(ii) The initiation of the redox transitions in the catalytic cycle of the Mn complex by short flashes of light was used to study structural changes at the metal site at room temperature. XANES spectra recorded within only $1 \mathrm{~s}$ on dark-adapted samples $\left(\mathrm{S}_{1}\right.$ state) revealed a shift of the $\mathrm{Mn} K$-edge to higher energies after one and two flashes (predominantly populating the $\mathrm{S}_{2}$ and $\mathrm{S}_{3}$ state). This effect is attributable to oxidation of the Mn complex ( $\mathrm{Mn}$ atoms plus ligands) (Ono et al., 1992; Roeloffs et al., 1996; Iuzzolino et al., 1998). The analysis of rapid-scan XANES spectra and of a series of time courses of flash-induced fluorescence changes consistently reveals distinct changes in the shape of the $\mathrm{Mn} K$-edge upon the $\mathrm{S}_{2} \Rightarrow \mathrm{S}_{3}$ transition. The different shapes of the $K$-edge spectra point to structural changes of the metal site upon the $S_{2} \Rightarrow S_{3}$ transition, a finding which is in line with results obtained by BioXAS measurements at low temperatures (see Robblee et al., 2001; Dau et al., 2001).

(iii) Laser-flash excitation of samples at the beamline results in synchronized progression of the Mn complex through its catalytic cycle. After each laser flash, the state changes of the Mn complex occur with halftimes in the $\mu$ s to ms range [halftimes of about $100 \mu \mathrm{s}$, $300 \mu$ s and $1 \mathrm{~ms}$ for the $\mathrm{S}_{1} \Rightarrow \mathrm{S}_{2}, \mathrm{~S}_{2} \Rightarrow \mathrm{S}_{3}$ and $\mathrm{S}_{3} \Rightarrow \mathrm{S}_{0}$ transition, respectively (Dekker et al., 1984)]. In the present study, we investigated the steady-state spectra reached within $20 \mathrm{~ms}$ after each flash. The temporal development of XAS spectra occurring within microseconds and milliseconds after the laser flash was not investigated. However, there are no principal impediments with respect to such measurements. In combination with a 'reference spectrum' collected for samples not previously exposed to laser flashes, the set of time courses of the fluorescence intensity measured for a set of X-ray excitation energies should allow for the construction of XAS spectra for each point in time. This sampling approach is not only applicable to the XANES region (as performed in these investigations) but also to the EXAFS range. On the basis of the sampling theorem it can be estimated that, by recording fluorescence transients at only 20-30 well selected excitation energies in the EXAFS range, absorberbackscatterer distances of up to $3.5 \AA$ may become 'visible'. In the near future, we will try to track the structural changes of the metal site which occur concomitantly to the redox reaction directly by timeresolved BioXAS with ms and, eventually, $\mu$ s resolution.

Financial support by the German BMBF (grant 05KS1KEA/6) and by the Alexander-von-Humboldt-Stiftung (for PP) is gratefully acknowledged. We thank the staff members at ID26 for excellent technical assistance.

\section{References}

Dau, H., Dittmer, J., Iuzzolino, L., Schiller, H., Dorner, W., Heinze, I., Sole, V. A. \& Nolting, H.-F. (1997). J. Phys. IV, 7, 607-610.

Dau, H., Iuzzolino, L. \& Dittmer, J. (2001). Biochim. Biophys. Acta, 1503, 24 39.

Dau, H., Iuzzolino, L., Dittmer, J., Dörner, W. \& Meyer-Klaucke, W. (1998). Photosynthesis: Mechanisms and Effects, edited by G. Garab, pp. 1327 1330. Dordrecht: Kluwer.

Dekker, J. P., Plijter, J. J., Ouwehand, L. \& van Gorkom, H. J. (1984). Biochim. Biophys. Acta, 767, 176-179.

Dittmer, J. \& Dau, H. (1998). J. Phys. Chem. B, 102, 8196-8200.

Dittmer, J., Iuzzolino, L., Dörner, W., Nolting, H.-F., Meyer-Klaucke, W. \& Dau, H. (1998). Photosynthesis: Mechanism and Effects, Vol. II, edited by G. Garab, pp. 1339-1342. Dordrecht: Kluwer.

Haumann, M., Grabolle, M., Neisius, T. \& Dau, H. (2002). FEBS Lett. 512, 116-120.

Haumann, M., Grabolle, M., Werthammer, M., Iuzzolino, L., Dittmer, J., Meyer-Klaucke, W., Neisius, T. \& Dau, H. (2001). Proceedings of the 12th International Congress on Photosynthesis. Brisbane: CIRSO. (http:// www.publish.csiro.au/ps2001/cf/home/index.cfm.)

Haumann, M. \& Junge, W. (1996). Oxygenic Photosynthesis: The Light Reactions, edited by D. Ort and C. Yocum, pp. 165-192. Dordrecht: Kluwer.

Hill, H. A. O., Sadler, P. J. \& Thomson, A. J. (1999). Metal Sites in Proteins and Models. Berlin: Springer.

Iuzzolino, L., Dittmer, J., Dörner, W., Meyer-Klaucke, W. \& Dau, H. (1998) Biochemistry, 37, 17112-17119.

Kok, B., Forbush, B. \& McGloin, M. (1970). Photochem. Photobiol. 11, $457-$ 475.

Liang, W., Roeloffs, T., Cinco, R., Rompel, A., Latimer, M. J., Yu, W., Sauer, K., Klein, M. P. \& Yachandra, V. K. (2000). J. Am. Chem. Soc. 122, 3399 3412.

Meinke, C., Solé, V. A., Pospíšil, P. \& Dau, H. (2000). Biochemistry, 39, 7033 7040.

Messinger, J., Robblee, J. H., Bergmann, U., Fernandez, C., Glatzel, P., Visser H., Cinco, R. M., McFarlane, K. L., Bellacchio, E., Pizarro, S. A., Cramer, S. P., Sauer, K., Klein, M. P. \& Yachandra, V. K. (2001). J. Am. Chem. Soc. 123, 7804-7820.

Ono, T., Noguchi, T., Inoue, Y., Kusunoki, M., Matsushita, T. \& Oyanagi, H. (1992). Science, 258, 1335-1337.

Penner-Hahn, J. E. (1999). Metal Sites in Proteins and Models: Redox Centres, edited by H. A. O. Hill, P. J. Sadler and A. J. Thomson, pp. 1-36 Heidelberg: Springer Verlag.

Pospíšil, P., Haumann, M., Dittmer, J., Solé, A. \& Dau, H. (2001). Proceedings of the 12th International Congress on Photosynthesis. Brisbane: CIRSO. (http://www.publish.csiro.au/ps2001/cf/home/index.cfm.)

Renger, G. (2001). Biochim. Biophys. Acta, 1503, 210-228.

Robblee, J. H., Cinco, R. M. \& Yachandra, V. K. (2001). Biochim. Biophys. Acta, 1503, 7-23.

Roeloffs, T. A., Liang, W., Latimer, M. J., Cinco, R. M., Rompel, A., Andrews, J. C., Sauer, K., Yachandra, V. K. \& Klein, M. P. (1996). Proc. Natl. Acad. Sci. USA, 93, 3335-3340. 
Schiller, H. \& Dau, H. (2000). J. Photochem. Photobiol. B Biol. 55, 138-144. Schiller, H., Dittmer, J., Iuzzolino, L., Dörner, W., Meyer-Klaucke, W., Sole, V. A., Nolting, H.-F. \& Dau, H. (1998). Biochemistry, 37, 7340-7350.

Scott, R. A. (2000). Physical Methods in Bioinorganic Chemistry - Spectroscopy and Magnetism, edited by L. Que Jr, pp. 465-504. Sausalito: University Science Books.
Signorato, R., Solé, V. A. \& Gauthier, C. (1999). J. Synchrotron Rad. 6, 176 178.

Solé, V. A., Gauthier, C., Goulon, J. \& Natali, F. (1999). J. Synchrotron Rad. 6, 174-175.

Yachandra, V. K. (1995). Methods in Enzymology, Vol. 246, Biochemical Spectroscopy, edited by K. Sauer, pp. 638-678. New York: Academic Press. 